\title{
Fiber Spectroscopy at Palomar Observatory
}

\author{
Donald Hamilton \\ Palomar Observatory, Robinson Laboratory of Astrophysics \\ California Institute of Technology
}

\begin{abstract}
A new faint-object spectrograph was designed and built around the capabilities of fiber optics. This instrument, the Norris Spectrograph, is designed exclusively for the Cassegrain focus of the Hale Telescope. There are 176 independently positionable fibers each with an aperture of $1.5 \mathrm{arcsec}$ in diameter. These 176 probes cover a field of view of about $20 \mathrm{arcmin}$ in diameter. The output ends of these 176 fibers are collimated with a spherical mirror, dispersed using a normal reflection grating, and focussed onto a Tektronix $2048 \times 2048$ CCD using all-transmissive optics. The peak overall efficiency of the instrument will be in excess of $10 \%$.
\end{abstract}

\section{INTRODUCTION}

A new multi-object spectrograph was recently designed and built at Palomar Observatory specifically around the characteristics of fiber optics. This instrument, the Norris Spectrograph, is an integrated fiber $x y$ stage and low-to-medium resolution spectrograph ( 0.1 to $1.6 \mathrm{~nm}$ resolution) optimizied for $380<\lambda<1000 \mathrm{~nm}$. Figure 1 shows one side of the completed spectrograph on its carrying cart. The grating rotator assembly, one of the guider modules, part of the $x y$ stage, and part of the CCD dewar can be seen. The upper cover has been removed so that part of the $x y$ stage can be seen. The spectrograph has 176 independently positionable fibers which are manipulated by a single pickup device riding on a rectilinear stage. Work on the instrument was begun in November 1987 and installed onto the telescope in June 1989. Many individuals contributed to the design and construction of the instrument including: Larry Blakee, Randy Baron, Michael Carr, John Cromer, Judy Cohen, Earle Emery, Lilo Hauck, Fred Harris, Greg van Idsinga, Chris Oke, and BeV Okè. A more detailed description of the instrument will be presented elsewhere ${ }^{1}$.

\section{OPTICAL CONFIGURATION}

The $f / 16$ focal ratio of the Cassegrain focus of the Hale Telescope is too slow for direct input into a fiber of nominal numerical aperture. In order to speed up the beam, a small quartz lens ( $2 \mathrm{~mm}$ diameter $\times 6 \mathrm{~mm}$ long) with a spherical surface of $3 \mathrm{~mm}$ radius of curvature changes the beam to $f / 7$ (in quartz). The light is directed at almost a right angle within this lenslet/prism by total internal reflection. Coupled to the output face of this lenslet/prism is the input end of the fiber.

The lenslet/prism is cemented into a brass envelope (hereafter referred to as the fiber head) which has a neck that is grabbed by the pickup device. The fiber heads are held rigidly in place on a ferrous plate of the $x y$ stage by rare-earth magnets which are afixed to the lower side.

Ideally, given typical seeing at Palomar, an entrance aperture of about one arcsec diameter would have maximized the contrast between an unresolved object and sky. However, since the Norris Spectrograph is intended for deep galaxy surveys, a diameter of 1.5 arcsec was deemed more appropriate. With an $f / 7$ beam, a fiber core diameter of 184 microns was needed to achieve this field of view. The core of the fiber is the field stop. The fibers 

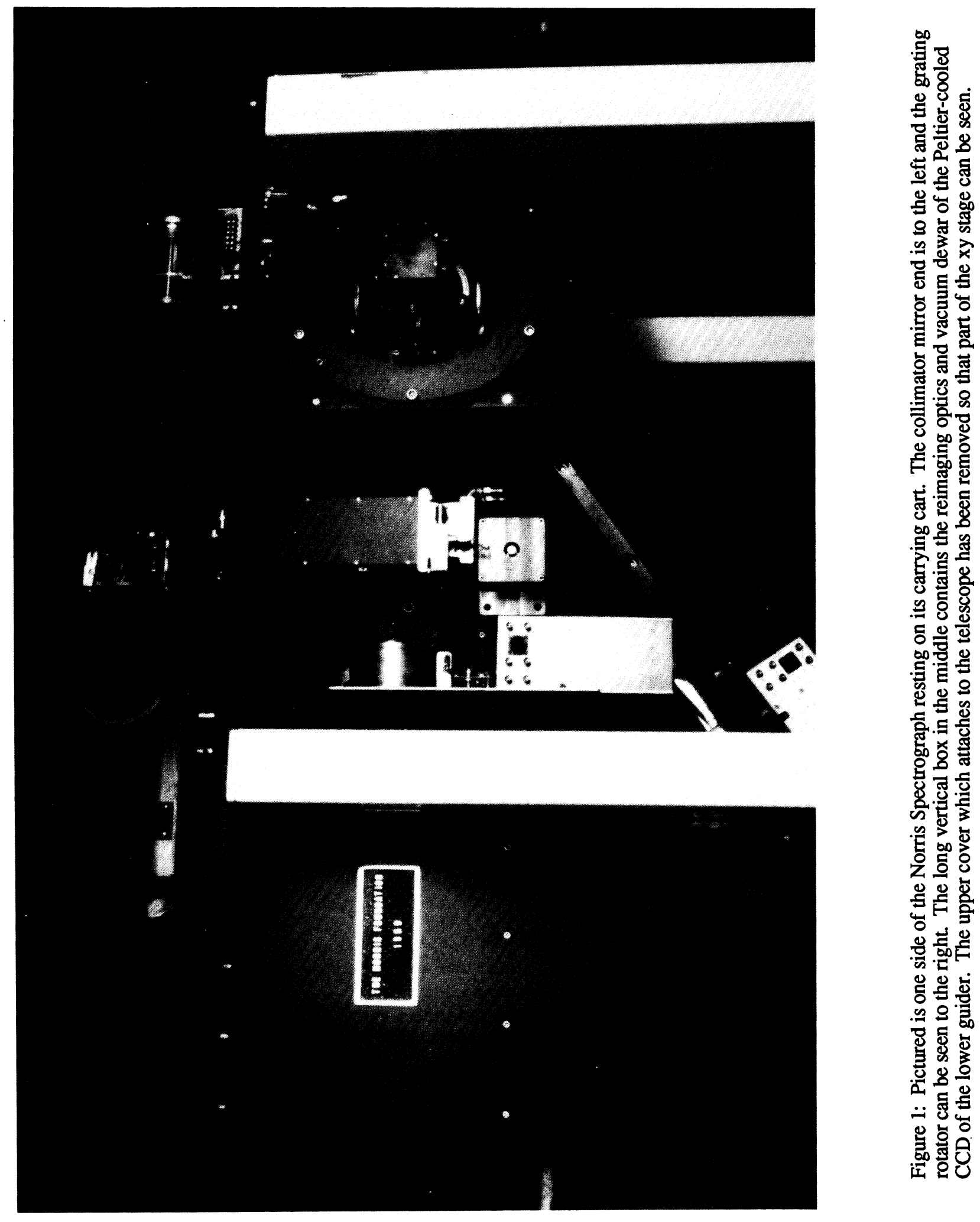
have a flat central profile of about one arcsec diameter.

Each end of the fiber is placed into a stainless steel hypodermic tube. The fiber is held within the tube by low shrinkage epoxy (EpoTek 353). After the epoxy was fully cured, the fiber and the stainless steel tube were polished until the fiber end was clean and flat. The stainless steel tube is cemented onto the fiber head.

Polymicro 'wet' fibers were used exclusively. Each of the 176 fibers is 2.2 meters long which is just sufficient to reach from the $x y$ stage to the fiber output (the 'slit') within the spectrograph. The fibers are brought straight off the stage perpendicular to the optical axis of the telescope. The stainless steel tube is fed through a guide which is free to move vertically at the end closest to the fiber head. The guide, made from plastic soda straws, is fastened at the other end and this acts as a pivot point. The bare fibers are bent through a radius of curvature of about $70 \mathrm{~mm}$ and fed underneath the stage into the side of the spectrograph below.

The output ends of the fiber are brought together side-by-side to form a 'virtual' slit at the curved focal plane of the collimator mirror. The slit assembly with an alignment fixture is pictured in Figure 2a. Some of the unfilled stainless steel tubes are seen in this picture. The mirror is a $150 \times 400 \mathrm{~mm}$ spherical mirror with a radius of curvature of $625 \mathrm{~mm}$. The mirror is coated with silver and overcoated with silicon monoxide for a peak reflectivity at $500 \mathrm{~nm}$. The dominant aberration of this mirror, spherical aberration, is small (45 microns) compared with the beam breadth of 184 microns at fiber output.

The reflection grating is located at the pupil of the collimator (or where a corrector would have been in a classical Schmidt camera). The gratings $(150 \times 203 \mathrm{~mm})$ are the same as those used with the Double Spectrograph ${ }^{2}$. The dispersed light from the grating is focussed using an all-transmissive optics design from Harland Epps. The detector used is a Tek $2048 \times 2048$ thick front-side illuminated CCD.

The Epps camera lens has a focal length and ratio of $305 \mathrm{~mm}$ and $\mathrm{f} / 1.34$ respectively. It has high throughput from 380 to $1000 \mathrm{~nm}$. The image size increases dramatically below $380 \mathrm{~nm}$ due to chromatic aberrations. The $r m s$ image size is 32 microns in white light. These final camera optics have yet to be installed into the instrument. With the exception of two fluorite elements, the Lick Optical Shop is fabricating the lenses. Temporary optics with the same focal length but with a clear aperture that is smaller by $40 \%$ than the final optics are currently in place and were used for testing purposes, although observations are possible with this configuration. The measured throughput using the existing low transmission camera optics is $>5.5 \%$ at $530 \mathrm{~nm}$. With the final camera optics in place the peak overall efficiency should be greater than $10 \%$.

The 2.0 demagnification of the optical system projects the output of the fiber to 90 microns on the CCD. The camera optics blur the image little so that there is an insignificant loss of resolution which is about three pixels (FWHM). Curvature of the spectrum from the length of the fiber slit implies less than a one pixel shift from center to end. The fibers are spaced approximately twelve pixels apart at the CCD detector. This spacing was intentional so that scattered light could be adequately modelled and subtracted.

The final camera lens will have mild sensitivity to temperature variations. The rms image size of the lens increases by about $10 \%$ when the temperature changes by about $10 \mathrm{C}$ from the nominal design values. This is due to the changes in curvatures and distances, but also the indices of refraction also are affected. The predominant change is from the two rather thick fluorite elements present in the Epps design.

\section{XY STAGE}

The stage onto which the 176 fibers are placed has an 'active' area of about $600 \times 600 \mathrm{~mm}$. The 'active' area on the sky amounts to a twenty arcmin diameter field. The stage consists of a ferrous plate (coated with Black Oxide) which is covered with a single layer of Teflon tape of 75 micron thickness. This tape protects and cushions both the stage plate and the magnet of the fiber head. A photograph of the completed $x y$ stage is given in Figure 3. The fibers are arranged in two opposing banks of 88 fibers each. At one end of the fiber heads (left side of Figure 3) and midway between the banks is a hole which is the position of the fixed (lower) guider.

The fiber heads are placed by a rectilinear $x / y$ axis system and a $z$ or pickup device. The $x$ axis is driven by a dual screw/motor system and the $y$ axis by a single drive. The dual drive system was originally envisioned so that fibers could be reliably placed irrespective of telescope position. However, in retrospect, a single drive would have sufficed, but a different design would be necessary.

The $x$ drive system consists of two high accuracy rails bracketing the 'active area' with two lead screws ( $3 \mathrm{~mm}$ pitch but with quadruple lead thread which reduces the effective pitch down to $12 \mathrm{~mm}$ ). Each of these lead screws is driven by a Compumotor AX 83-135. microstepping motor. The $y$ axis system is similarly configured but with 

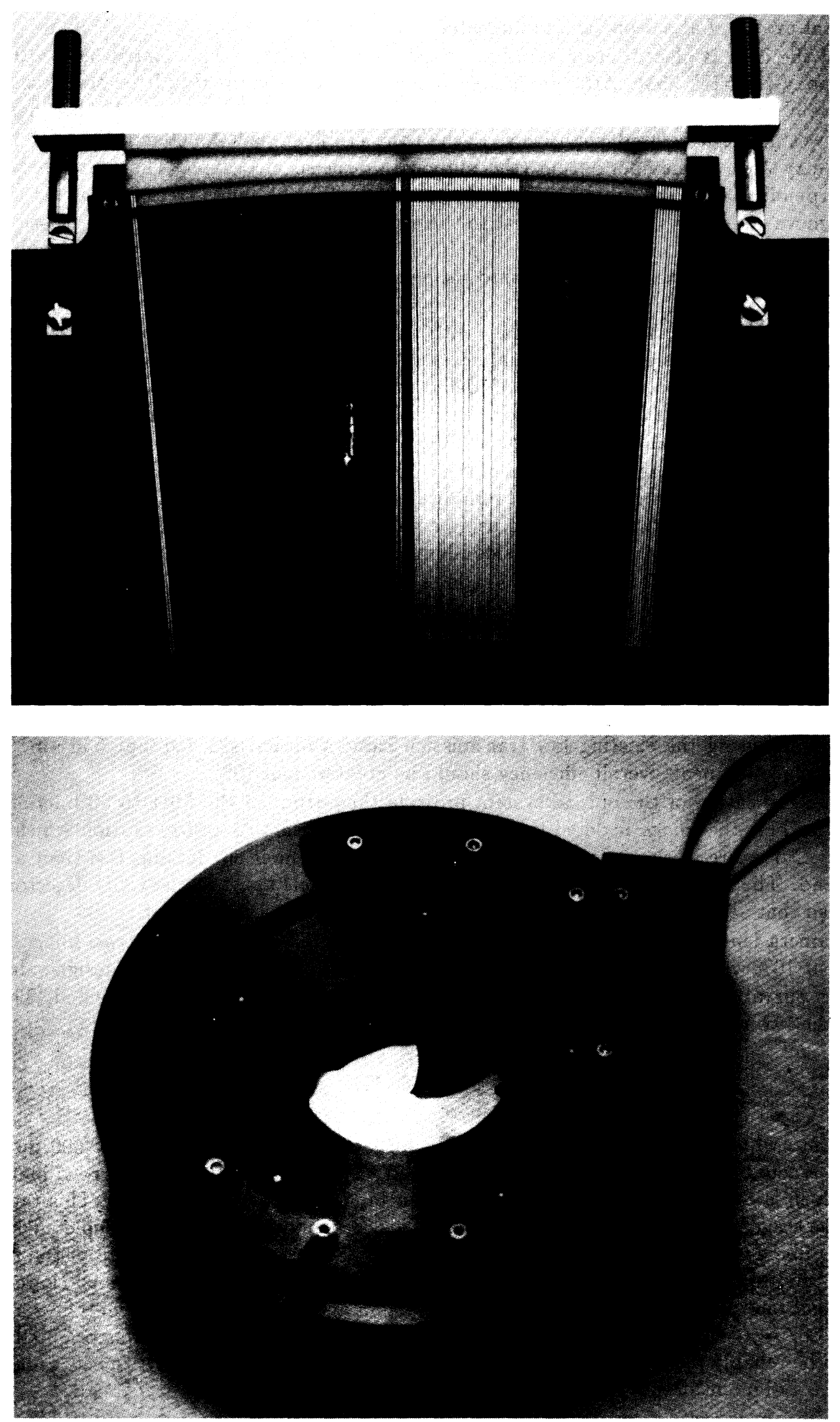

Figure 2: Pictured are selected parts of the Norris Spectrograph: 2a) the fiber slit assembly which holds the output ends of the fibers along the curved focal plane of the collimator mirror; and 2b) the shutter assembly for the Tektronix 2048 CCD. 


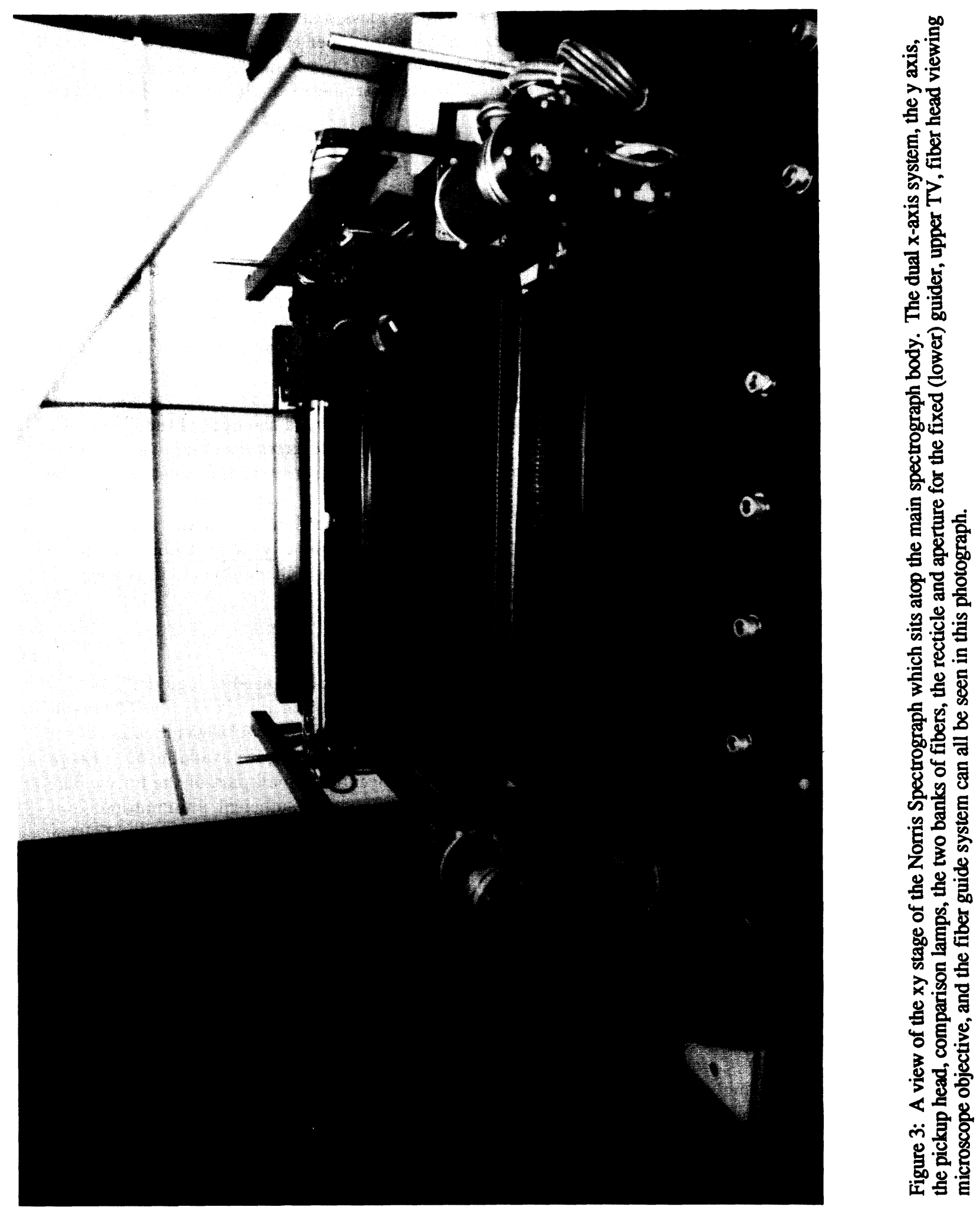


a single drive and a Compumotor $\mathrm{AX}$ 83-93 motor. Compumotor microstepping motors were used throughout the spectrograph for their communications and programming ease (only ASCII input over a serial line).

Each of the three axes involved (two $x$ and one $y$ ) is encoded using a glass scale incremental linear encoder. The encoder steps are two microns apart which translates into two microsteps per encoder step. The measured accuracy of the $x y$ stage is better than ten microns. This translates into 0.04 arcsec at the Cassegrain focus of the Hale Telescope. The limiting factor in placing the fibers will be the astrometry of the objects and not the mechanics of the $x y$ stage.

\section{FIBER PICKUP MECHANISM}

Riding on the $y$ axis which rides upon the $x$ axes is the fiber pickup or $z$ head. This entirely mechanical device grabs the neck of the fiber head using a three point collet. The pickup device consists of a pinion which is attached to the shaft of a Compumotor AX 57-51 motor. This pinion drives a rack attached to a vertical sliding assembly (sleeve) which holds the collet. The high precision collet ( $\sim 5$ microns) was specially manufactured by Hardinge to our tolerances. The collet movement is actuated by a Ledex rotary solenoid and by spring loading the connecting arm the default state is closed. The collet and its actuating mechanism are placed within a sleeve and are completely free to rotate.

The procedure to pick up a fiber is to first energize the solenoid and hence open the collet chuck. The stepper motor is then rotated a known number of microsteps and the collet will have enveloped the neck of the fiber head. The power to the solenoid is removed and the collet closes. The stepper motor reverses direction and the fiber head is lifted from the ferrous plate. The fiber head can be off as much as 100 microns and the collet will still be able to collect it.

In order to sense the presence of a fiber, an infrared LED and diode are placed such that the line between the two will be intersected by a fiber head inside the collet. If in the extremely unlikely event a fiber should be lost, there is a hunt and peck mode of the pickup device which can retrieve a fiber under some circumstances.

\section{GUIDING AND FIELD ACQUISITION}

There are two CCD cameras, referred to as upper and lower, either of which can be used as a guider TV. The upper camera is a commercially available uncooled, variable integration high resolution Pulnix camera. This unit is attached next to the pickup device and moves with it. There are focal reduction optics which yield a scale of 0.1 arcsec per pixel. Both on-chip integration and coaddition of frames in a video memory are available to enhance detection. The upper camera is uplooking although there is a prism which occults a small part of the beam. The prism permits viewing of a fiber head by a microscope objective, but only if there is sufficient illumination.

The lower camera system is a three-stage Peltier cooled TI 4849 virtual phase CCD which can be used either in a frame-transfer or full frame mode. The standard Palomar CCD electronics are used ${ }^{3}$. The CCD is controlled and the frames collected by an AMIGA 1000 68000-based computer. Programs for guiding and field acquisition were written in C. There are reimaging optics upstream from the CCD which consist of a $150 \mathrm{~mm}$ focal length $f / 2.8$ collimating lens and a $28 \mathrm{~mm}$ focal length camera lens. The resultant scale is $0.3 \operatorname{arcsec}$ per 22 micron pixel.

\section{INSTRUMENT CONTROL}

All of the functions of the spectrograph except for the lower guider are controlled by a $68020 \mathrm{VME}$-bus based computer (FORCE CPU 29). Communication between the spectrograph and the computer (located on the mezzanine floor of the 200-inch dome) are done through two serial lines. At either end of these lines there is an asynchronous local time-division multiplexer which can handle up to twelve RS-232 lines (without handshaking).

The FORCE computer contains a small Winchester disc and after every setup of the $x y$ stage the current positions of all the fibers are written to disc. In addition, there is battery backup to the CPU memory so if there should occur a power failure, a full recovery is trivial.

The computer controls all functions of the spectrograph. Simple commands rotate the grating; open and close the trap door; clamp and unclamp the grating brake; clamp and unclamp the $x y$ stage brakes; and move each of the three axes $x, y$, and $z$. 


\section{ECHELLE MODE}

A unique feature of the Norris Spectrograph was to have been a high resolution mode using an echelle grating. Originally a single order was to have been isolated using a narrow passband filter in front of the existing fiber slit. This design evolved into a full echelle mode with the use of a twice-through quartz prism cross-disperer so that the entire spectrum from 380 to $980 \mathrm{~nm}$ could be observed at once.

The echelle grating was to have been placed immediately behind the normal grating which would have been replaced with a 60 degree fused quartz prism. The echelle mode was to have been accomplished by feeding the Epps camera in reverse with fibers and hence the optics would have been in autocollimation. Two smaller core diameter fibers than used with the regular spectrograph would have fed the camera lens. Right angle quartz prisms (basically the lenslet/prism mentioned above with the spherical surface cut off) would have obviated the need for bending the fibers.

The output end of the two fibers would have been at the prime focus of an inverse Cassegrain system where a small ( $\sim$ one cm diameter) hyperbolic mirror would have directed the beam in reverse through the Epps camera. Cemented to the Cassegrain secondary would have been an achromatic doublet. The rms spot size would have been about 48 microns $(\sqrt{2}$ times the spot size for the single pass camera). The Cassegrain secondary and its doublet would have been cemented directly to the field flattener of the camera lens. This element would be removable so that the spectrograph modes could have been interchanged straightforwardly.

Using a prism instead of a grism as a cross-disperser allowed for a more uniform inter-order spacing and was also a more efficient use of CCD real estate. The $\mathrm{R} 2$ echelle would have had 79 lines per $\mathrm{mm}$. The resultant resolution would have been $0.036 \mathrm{~nm}$ (4 pixels). The resolution is twice the norm due to the chromatic aberrations of the Epps lens used in autocollimation and to the size of the fiber used.

Within this configuration the spectrum from 380 to $980 \mathrm{~nm}$ would have been observable at once. This area would have taken up only' about one half of the Tektronix 2048 CCD and hence two fibers could be observed simultaneously. Two fibers would have permitted simultaneous sky and object measurements. Beam switching between these fibers would have averaged out residual or variable relative throughputs between the fibers.

A possible problem which was not investigated was scattered light. No ghost analysis was performed as a non-technical reason stopped the echelle mode of the Norris Spectrograph altogether.

\section{MISCELLANEOUS}

The entire spectrograph (stage and optics) are enclosed and the air inside is purged with boil-off gas from the liquid nitrogen reservoirs of the CCD dewars. There is an entrance window to the instrument made out of fused quartz and polished to a few tens of waves by Custom Optics of Tucson, AZ. This window is coated with a single layer of magnesium fluoride for maximum transmittance at $450 \mathrm{~nm}$. Protecting the window is a trap door which folds up into the Cassegrain tube of the 200-inch telescope.

Comparison arc lamps of neon, argon, mercury, and an incandescent lamp have been placed around the periphery of the $x y$ stage. These lamps illuminate a reflective screen on the underside of the trap door. This arrangement provides adequate illumination of all the fibers. The fibers can only see light that is reflected off the the screen due to the necks of the fiber heads acting as a light baffle.

In order to ensure that fibers can be placed at the correct positions of objects (assuming error-less coordinates), a procedure has been developed for modelling and mapping the distortions of the focal plane of the Cassegrain focus. Stars in a bright open cluster are observed serially with the upper TV system while guiding the telescope with the lower guider. The predicted (or first order) positions of the stars are determined from accurate photographic astrometry and the errors are sufficiently small that non-linear least squares fitting techniques may be appropriately used.

Setup software has been written to take a list of objects (say from scans of photographics plates) and extract a sub-sample of these objects which will fit within a field-of-view of the Norris Spectrograph. This sub-sample list is sent through a sequence of programs to correct for precession and proper motion (if known) and to correct for atmospheric differential refraction. The objects of the corrected list are then matched with fiber heads. There is an automatic assignment of fibers to objects (with and without matching sky fibers) and an assignment program in which the operator can assign fibers to objects manually. These programs were originally written in FORTH, but were later translated into FORTRAN for the general user public. 
Due to the large size of the Tektronix $2048 \mathrm{CCD}$, no affordable high quality shutter could be found. The maximum aperture for the Uniblitz line of shutters is only $55.0 \mathrm{~mm}$. A large aperture $(100 \mathrm{~mm})$ shutter was developed from an iris diaphragm used in an areal camera lens ${ }^{4}$. The shutter is actuated by a dual solenoid system. The response time is approximately 0.1 secs. The shutter is pictured in Figure $2 \mathrm{~b}$.

The detector used in the spectrograph is a Tektronix $2048 \times 2048$ thick front-side illuminated CCD. This CCD has a peak quantum efficiency of $32 \%$ at $650 \mathrm{~nm}$. The CCD is operated at $-96 \mathrm{C}$ which is warmer than other CCD's at Palomar. This warmer operating temperature was necessary as temperature senstive traps were present in the serial output register which precluded respectable flat-fielding ${ }^{5}$.

In order to achieve high precision flat-fielding with fibers the output image of the fiber is moved perpendicular to the dispersion. This dithering is accomplished by rocking the collimator mirror by a small amount. The projected motion onto the CCD is altered slightly by constantly changing the angular velocity of a motor. The variable acceleration results is a uniform flat-field over ten pixels.

\section{ACKNOWLEDGEMENTS}

This instrument was fully funded by The Norris Foundation. Their interests and contributions are most gratefully acknowledged. Jim Westphal was most helpful in allowing use of his lab during the final construction stages of the spectrograph.

\section{REFERENCES}

1. D. Hamilton et al., "A Multi-Object Fiber Spectrograph for the Hale Telescope," in preparation.

2. J. B. Oke and J. E. Gunn, "An Efficient Low- and Moderate-Resolution Spectrograph for the Hale Telescope," Pub. A. S. P., 94, p. 586, Jun. 1982.

3. J. E. Gunn et al., "The Palomar Observatory CCD Camera," Pub. A. S. P., 99, p. 518, June 1987.

4. D. Hamilton et al., "An In-expensive Shutter for Large-format CCD's," in preparation.

5. D. Hamilton et al., "Testing of Tektronix CCD's at Palomar Observatory," in preparation. 